\title{
Cooperative Passenger Inflow Control in Urban Mass Transit Network with Constraint on Capacity of Station
}

\author{
Jianyuan Guo, ${ }^{1}$ Limin Jia, ${ }^{2}$ Yong Qin, ${ }^{2}$ and Huijuan Zhou $^{3}$ \\ ${ }^{1}$ School of Traffic and Transportation, Beijing Jiaotong University, Beijing 100044, China \\ ${ }^{2}$ State Key Laboratory of Rail Traffic Control and Safety, Beijing Jiaotong University, Beijing 100044, China \\ ${ }^{3}$ Beijing Key Lab of Urban Intelligent Traffic Control Technology, North China University of Technology, Beijing 100144, China
}

Correspondence should be addressed to Limin Jia; lmjiaedu@126.com

Received 24 July 2015; Revised 6 October 2015; Accepted 12 October 2015

Academic Editor: Seenith Sivasundaram

Copyright (C) 2015 Jianyuan Guo et al. This is an open access article distributed under the Creative Commons Attribution License, which permits unrestricted use, distribution, and reproduction in any medium, provided the original work is properly cited.

\begin{abstract}
In urban mass transit network, when passengers' trip demands exceed capacity of transport, the numbers of passengers accumulating in the original or transfer stations always exceed the safety limitation of those stations. It is necessary to control passenger inflow of stations to assure the safety of stations and the efficiency of passengers. We define time of delay (TD) to evaluate inflow control solutions, which is the sum of waiting time outside of stations caused by inflow control and extra waiting time on platform waiting for next coming train because of insufficient capacity of first coming train. We build a model about cooperative passenger inflow control in the whole network (CPICN) with constraint on capacity of station. The objective of CPICN is to minimize the average time of delay (ATD) and maximum time of delay (MTD). Particle swarm optimization for constrained optimization problem is used to find the optimal solution. The numeral experiments are carried out to prove the feasibility and efficiency of the model proposed in this paper.
\end{abstract}

\section{Introduction}

More and more passengers travel by urban mass transit (UMT) because UMT is rapid, punctual, and green. With the increasing number of passengers, the capacity of urban mass transit network (UMTN) cannot satisfy the trip demands of passengers. There are lots of passengers who queue and wait because of insufficient capacity of trains [1]. Dangers are brought while there are too many waiting passengers in stations. In the cities in China, such as, Beijing, Guangzhou and Shanghai, the UMT corporations usually control passenger inflow in stations where there are too many waiting passengers to assure safety $[2,3]$.

The approaches of passenger inflow control include controlling passenger inflow in a special station [4], in two stations [5], in stations on a special line [6], or in stations on different lines [7]. In the studies above, the model of controlling passenger inflow in a special station, two stations, and stations on a special line has been described accurately and solved by some methods. Controlling passenger inflow of stations in different lines is described in a qualitative way and there is no model and no effective method.

In UMTN, many trips involve at least one transfer [8]. Transferring passenger takes a great proportion of waiting passengers in transfer stations. The number of waiting passengers cannot be controlled under safety limitation only by controlling inflow of this transfer station. It is necessary to limit the inflow of original station which constitutes the transferring passengers and waiting passengers in this transfer station. So the cooperative inflow control involving stations in the whole network is needed to reduce the pressure of transfer station and improve the safety and efficiency of passengers' trips. This will be described in detail in Section 2.1.

Thus we build model to describe cooperative passenger inflow control in the whole network (CPICN). This model and the solving method will provide theoretic support for planning control measure in real operation.

The rest of this paper is organized as follows. Section 2 analyzes the necessity and evaluation criteria of CPICN. 


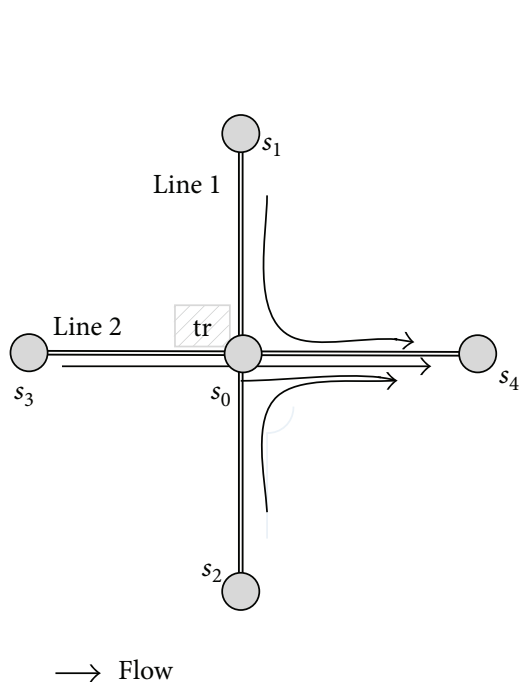

(a)

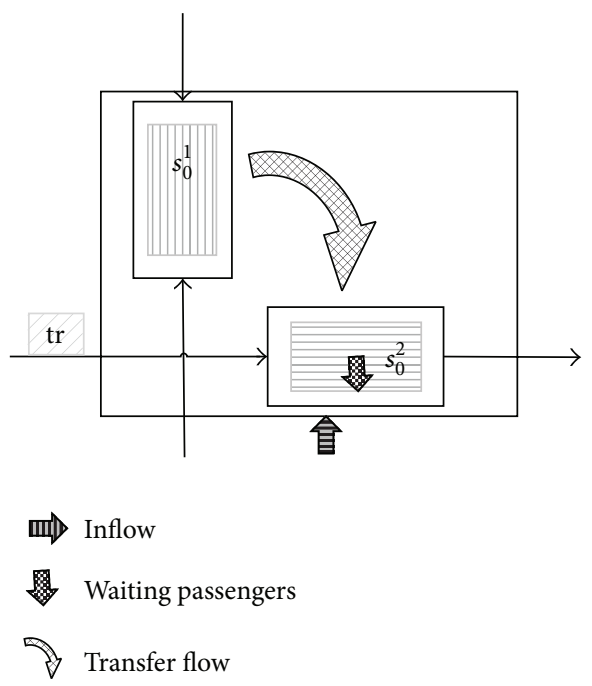

(b)

FIGURE 1: Compositions of waiting passengers in transfer station.

Section 3 builds the model, including assumption, objective, and constraints. Section 4 solves the model by PSO algorithm. Section 5 carries out numerical experiments to prove the feasibility and effectiveness of this model and solving method. Section 6 summarizes the contributions in this paper and puts forward the further research.

\section{Analyzing CPICN}

2.1. The Necessity of CPICN. A simple network is constructed as shown in Figure 1 to illustrate the composition of waiting passengers in transfer station. Figure 1(b) describes some detail in Figure 1(a). In the simple network there are 5 stations which are $s_{0}, s_{1}, \ldots, s_{4} . s_{0}$ is a transfer station. We notate the waiting area (such as platform) in $s_{0}$ on line 1 and line 2 as $s_{0}^{1}$ and $s_{0}^{2}$, respectively. Denote $\mathrm{PL}_{0}^{j}$ as the safety limitation of $s_{0}^{j}(j \in\{1,2\})$ and $W_{0}^{j}$ as the number of waiting passengers in $s_{0}^{j}$. When $W_{0}^{j}>\mathrm{PL}_{0}^{j}, s_{0}^{j}$ is not safe and it is necessary to control number of waiting passengers to satisfy $W_{0}^{j} \leq \mathrm{PL}_{0}^{j}$ to assure the safety of $s_{0}$.

Suppose train $\operatorname{tr}$ departs from $s_{3}$, goes to $s_{4}$, and passes by $s_{0}^{2}$. The passengers who wait for tr at $s_{0}^{2}$ consist of passengers who depart from $s_{1}$ or $s_{2}$ and go to $s_{4}$ and passengers who enter $s_{0}$ and depart from $s_{0}^{2}$. Here denote $W_{i-0}^{j}(i \neq 0)$ as the passengers who enter $s_{i}(i \neq 0)$, alight from train at $s_{0}^{1}$ and wait $\operatorname{tr}$ in $s_{0}^{2}$. When the number of transferring passengers satisfies $\sum_{i=2}^{3} W_{i-0}^{j}>\mathrm{PL}_{i-0}^{j}$, even though there are no passengers who enter $s_{0}$ and no passengers who get on tr at $s_{3}$, the safety of $s_{0}$ cannot be guaranteed because of too many waiting passengers. Under this condition, it is necessary to limit the number of inflow at $s_{i}(i \neq 0)$ to assure $W_{0}^{j} \leq \mathrm{PL}_{0}^{j}$.

According to above illustration, it is necessary to use method of CPICN to assure safe of transfer station in real network when there are too much transferring passengers.

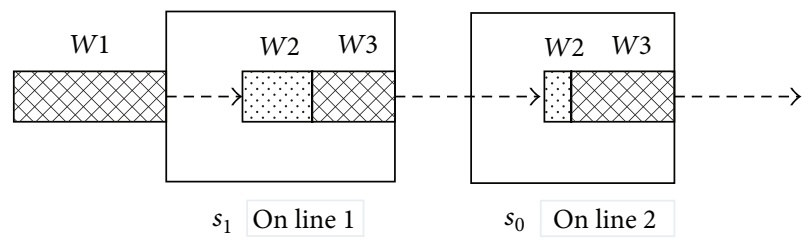

FIgURE 2: Example of compositions of waiting time.

2.2. How to Evaluate Solution of CPICN. Passenger inflow control can change the number of passengers waiting outside or inside of stations. The waiting time of passengers can be changed along with it. The waiting time of a passenger may consist of several parts shown in Figure 2. These waiting parts can be classified into three types: $W 1, W 2$, and $W 3$. $W 1$ represents the waiting time outside of station caused by inflow control, $W 2$ represents the waiting time used to wait for the first coming train, and $W 3$ represents the waiting time used to wait for the next coming train which can be boarded because of insufficient capacity of the first coming train. $W 2$ is inevitable for a trip. $W 1$ and $W 3$ are extra delay for passengers' trip, which can be changed and controlled by inflow control. Here we name the sum of $W 1$ and $W 3$ as time of delay (TD).

Denote TDOC $_{p}$ as the time used by passenger $p$ to wait outside of station because of inflow control, which corresponds to $W 1$. Denote $\mathrm{TDIO}_{p}$ as the extra time used to wait for next train that can be boarded, which corresponds to $W 3$. TD for a special passenger $p$ can be described as follows:

$$
\mathrm{TD}_{p}=\mathrm{TDOC}_{p}+\mathrm{TDIO}_{p}
$$

Inflow control leads to less TD for some passengers while more TD for some other passengers. So we use ATD to evaluate the solution which represents average TD. It is computed according to (2), where PA is the set of passengers 
in whole network and PAC is the number of passengers in PA. For the fixed transport capacity of network, if ATD is less, more passengers are served and the control solution is better

$$
\mathrm{ATD}=\frac{\sum_{p \in \mathrm{PA}} \mathrm{TD}_{p}}{\mathrm{PAC}} .
$$

Moreover, it exists that some passengers have longer TD than others. If $\mathrm{TD}_{p}$ is much longer than $\mathrm{TD}_{p^{\prime}}\left(p^{\prime} \in \mathrm{PA}-\{p\}\right)$, it is unfair for passenger $p$. So we define MTD to represent the longest $\mathrm{TD}_{p}$, which can be described as (3). The solution is better while MTD is less

$$
\mathrm{MTD}=\max \left(\mathrm{TD}_{p}\right), \quad p \in \mathrm{PA} .
$$

Thus, the general efficiency and individual interest are both considered. The solution of CPICN is needed to satisfy minimum ATD and MTD.

\section{Building Model of CPICN}

3.1. Assumptions of Model. To simplify the problem and highlight the key conceptions, some assumptions are given as follows.

(1) The time-dependent O-D trip demand is known and discretized into time segments. It is a uniform random passenger arrival distribution in a time segment $\Delta \mathrm{ts}$.

(2) The control solution is time varying for every station.

(3) Passengers queue and board trains according to firstin-first-out discipline.

(4) All passengers who enter transit network will reach their planned destination and will not give up the trip at midway.

(5) All transit trains have fixed capacity and operate precisely according to specified timetables.

(6) Capacity of platform is used as the capacity of station supplied for waiting passengers.

(7) In a transfer station, different lines use different platforms.

3.2. Model of CPICN. According to Section 2.2, the objective of CPICN should minimize ATD and MTD, which is described as (4a). The constraints of model encompass capacity of station, capacity of train, O-D demand, and flow conservation, which are described as (4b)-(4e)

$$
\begin{array}{ll}
\min & \operatorname{ATD} \wedge \min \mathrm{MTD} \\
\text { S.T. } & W_{i}^{j} \leq \mathrm{PL}_{i}^{j} \\
& \operatorname{tp}_{\mathrm{tr}} \leq \mathrm{tc}_{\mathrm{tr}} \\
& c_{i}^{\mathrm{ts}} \leq w_{i}^{\mathrm{ts}} \\
& \operatorname{tp}_{\mathrm{tr}}^{i}-\mathrm{al}_{\mathrm{tr}}^{i}+\mathrm{ab}_{\mathrm{tr}}^{i}=\mathrm{tp}_{\mathrm{tr}}^{i+1} .
\end{array}
$$

Equation (4b) represents that the number of waiting passenger in $s_{i}^{j}$ should not exceed $\mathrm{PL}_{i}^{j}$.
Equation (4c) represents that the number of passengers aboard train $t r$ should not exceed the capacity of $t r$, where $t p_{t r}$ is denoted as number of passengers aboard $\mathrm{tr}, \mathrm{tc} \mathrm{tr}_{\mathrm{tr}}$ as capacity of tr.

In $(4 \mathrm{~d}), c_{i}^{\mathrm{ts}}$ is the maximum number of passengers allowed to enter station $s_{i}$ under control in time segment ts, and $w_{i}^{\text {ts }}$ is the number of passengers who want to enter station $s_{i}$ in this time segment. $w_{i}^{\text {ts }}$ can be expressed as (5), where $a_{i}^{\text {ts }}$ is the number of arrival passengers who arrive at $s_{i}$ in time segment ts, and ts -1 is the time segment before ts

$$
\begin{aligned}
& w_{i}^{\mathrm{ts}}=a_{i}^{\mathrm{ts}}+w_{i}^{\mathrm{ts}-1}-c_{i}^{\mathrm{ts}-1}, \quad \text { ts }>1, \\
& w_{i}^{1}=a_{i}^{1} .
\end{aligned}
$$

Equation (4e) corresponds to assumption (4a), (4b), (4c), (4d), and (4e), where $\mathrm{tp}_{\mathrm{tr}}^{i}$ is the number of passengers on $\operatorname{tr}$ when $\operatorname{tr}$ arrives at station $s_{i}$. $\mathrm{al}_{\mathrm{tr}}^{i}$ and $\mathrm{ab}_{\mathrm{tr}}^{i}$ represent the number of passengers alighting from tr and boarding tr at $s_{i}$, respectively. $\mathrm{tp}_{\mathrm{tr}}^{i+1}$ represents the number of passengers on $\mathrm{tr}$ when $\operatorname{tr}$ arrives at next station $s_{i+1}$.

\section{Solution Procedure}

4.1. Method Used to Solve Model. According to assumption (2), the solution of model can be expressed as in (6), where $n$ is the number of stations and $m$ is the number of time segments (the solution of model):

$$
C=\left[\begin{array}{ccc}
c_{1}^{1} & \cdots & c_{1}^{m} \\
\vdots & \vdots & \vdots \\
c_{n}^{1} & \cdots & c_{n}^{m}
\end{array}\right]
$$

The maximum numbers of passengers allowed to enter station in different time segments of different stations influence each other for the constraints of model. Because of the complexity, particle swarm optimization (PSO) for constrained optimization problem is chosen to solve the model.

Particle swarm optimization (PSO) originally was developed by Eberhart and Kennedy in 1995 [9], which belongs to a class of methods known as evolutionary computation. It hypothesizes that there are $m$ particles in the $\mathrm{D}$ dimensions' space. Fitness function used to evaluate particles in space. For the particle $i$ of iterative $t+1$ generation, the position and velocity of particles are adjusted according to the position and velocity in the previous $t$ generation, as follows:

$$
\begin{gathered}
v_{i d}(t+1)=v_{i d}(t)+c_{1} r_{1} \times\left(p_{i d}(t)-x_{i d}(t)\right) \\
+c_{2} r_{2}\left(p_{g d}(t)-x_{i d}(t)\right), \\
x_{i d}(t+1)=x_{i d}(t)+v_{i d}(t+1),
\end{gathered}
$$

where $x_{i d}$ and $v_{i d}$ are position and velocity of particle $i$ in dimensionality $d$. $c_{1}$ and $c_{2}$ are weight coefficients of the particle individual and $r_{1}$ and $r_{2}$ are two random functions whose values are between 0 and 1 . $p_{i d}$ represents the previous best local position of the particle $i$. $p_{g d}$ represents the best global position found up by the whole swarm. 
An important improvement in the basic PSO is the use of an inertia factor $\omega[10]$ that multiplies the velocity term in (7), as shown in (9). In general, $\omega$ starts large and is decremented from $w_{\text {ini }}$ to $w_{\text {end }}$ during the evolution of the algorithm as shown in (10), where Gk is the largest generation of algorithm

$$
\begin{aligned}
v_{i d}(t+1)= & \omega v_{i d}(t)+c_{1} r_{1} \times\left(p_{i d}(t)-x_{i d}(t)\right) \\
& +c_{2} r_{2}\left(p_{g d}(t)-x_{i d}(t)\right) \\
\omega(t)= & \left(w_{\text {ini }}-w_{\text {end }}\right) \times \frac{(\mathrm{Gk}-t)}{\mathrm{Gk}}+w_{\text {end }} .
\end{aligned}
$$

Clerc and Kennedy [11] proposed an alternative version of PSO in which the convergence factor is used, and the velocity adjustment is given as

$$
\begin{aligned}
v_{i d}(t+1)= & k\left[\omega v_{i d}(t)+c_{1} r_{1} \times\left(p_{i d}(t)-x_{i d}(t)\right)\right] \\
& +c_{2} r_{2}\left(p_{g d}(t)-x_{i d}(t)\right)
\end{aligned}
$$

where $k$ is the convergent factor, which is computed as (12), where $\phi=c_{1}+c_{2}>4$

$$
k=\frac{2}{\left|2-\phi-\sqrt{\left|\phi^{2}-4 \phi\right|}\right|} .
$$

PSO for constrained optimization problem finds optimal location of particle which has best fitness and satisfies the constraints. The approaches focus on several ways, such as adding penalty function to fitness function [12], valuing the infeasible solution by constraints violation [13, 14], adapting PSO parameters according to constraints violation $[15,16]$, generating new feasible solution [17], and adjusting parameters to make infeasible solution become feasible [18].

Referencing $[13,18]$, when adjusting infeasible solution to feasible, the rule updating best solution is described as follows.

Rule 1. (1) Any feasible solution is preferred to any infeasible solution.

(2) Between two feasible solutions, the one having smaller ATD and smaller MTD is preferred.

(3) Between two infeasible solutions, the one having smaller constraint violation is preferred.

Moreover, infeasible solution is valuable for finding optimal solution. Some researchers proposed "Do not kill unfeasible individuals" [19] and "Unfeasible individuals may well contain information valuable enough for them to be preserved" [20]. So we divide particles into two subpopulations. The best solution in population 1 must be feasible, and the best solution in population 2 is not constrained by feasible space. Two populations cross obeying fix rate $\rho$.

The rules updating best solution of population $1\left(\mathrm{po}_{1}\right)$ and population $2\left(\mathrm{po}_{2}\right)$ are described as Rules 2 and 3, respectively.

Rule 2. (1) Any feasible solution is preferred to any infeasible solution.

(2) Between two feasible solutions, the one having smaller $\mathrm{ADT}$ and smaller MDT is preferred.
TABLE 1: Time varying O-D demand.

\begin{tabular}{lcccc}
\hline $6: 30-7: 00$ & $7: 00-7: 30$ & $7: 30-8: 00$ & $8: 00-8: 30$ & $8: 30-9: 00$ \\
\hline 2100 & 4200 & 6300 & 4200 & 2100 \\
\hline
\end{tabular}

Rule 3. The one having smaller ADT and smaller MDT is preferred.

When crossing, velocity of particle $i$ is computed according to (13), where $j$ is the index of population, such as $p_{g d}^{1}(t)$ is the best solution in $\mathrm{po}_{1}$ and $p_{g d}^{2}(t)$ is the best solution in $\mathrm{po}_{2}$

$$
\begin{aligned}
v_{i d}^{j}(t+1)= & k\left[\omega v_{i d}^{j}(t)+c_{1} r_{1} \times\left(p_{i d}^{j}(t)-x_{i d}^{j}(t)\right)\right] \\
& +c_{2} r_{2}\left(p_{g d}^{2-j}(t)-x_{i d}^{j}(t)\right), \quad j \in\{1,2\} .
\end{aligned}
$$

Adjust infeasible solution to feasible solution referencing [18] when the rate of infeasible solution exceeds up limit of rIF. rIF of population po is computed as (14), where QIF $_{\text {po }}$ is the number of infeasible solutions in po and $Q_{p o}$ is the size of po. The up limit of rIF is $\beta$ in this paper

$$
\mathrm{rIF}_{\mathrm{po}}=\frac{\mathrm{QIF}_{\mathrm{po}}}{\mathrm{Q}_{\mathrm{po}}}
$$

4.2. Solving Procedure. Based on the above method, the flow for solving procedure is shown in Figure 3, where network loading includes load lines, stations, transfer relationship, and schedule.

In the procedure, fitness function is computed by simulation referencing [21-23]. During simulating, set the limit of inflow according to location of particle, update the number of passengers outside of stations, in stations, and on the trains with the train arriving and departing, and compute MTD and ATD at the end of simulating.

\section{Numerical Experiments}

5.1. Background of Experiments. In order to demonstrate the key features of CPICN, experiments are carried out on a simple network with many-to-one OD flow shown in Figure 1. The inflows of $s_{0}, s_{1}, s_{2}, s_{3}$ are the same, which are time varying as shown in Table 1 .

The safe limit of platform is 1800 . The capacity of train is 1856 . The headway of trains is 180 seconds and the time holding on station of trains is 30 seconds. The running time between two stations of trains is 300 seconds. The transfer time in transfer station is 120 seconds.

5.2. Effectiveness of Algorithm. Algorithms are programed by Java and compiled by JDK 7.25 for Windows x64 in Eclipse 10. Experiments are tested on a personal computer with an Intel Core i5, $2.50 \mathrm{GHz}$ CPU, and $4 \mathrm{~GB}$ RAM. The parameters of algorithms are set as shown in Table 2.

The algorithm in this paper is named as Algorithm A. The algorithm proposed by Sun et al. [18] is named as 


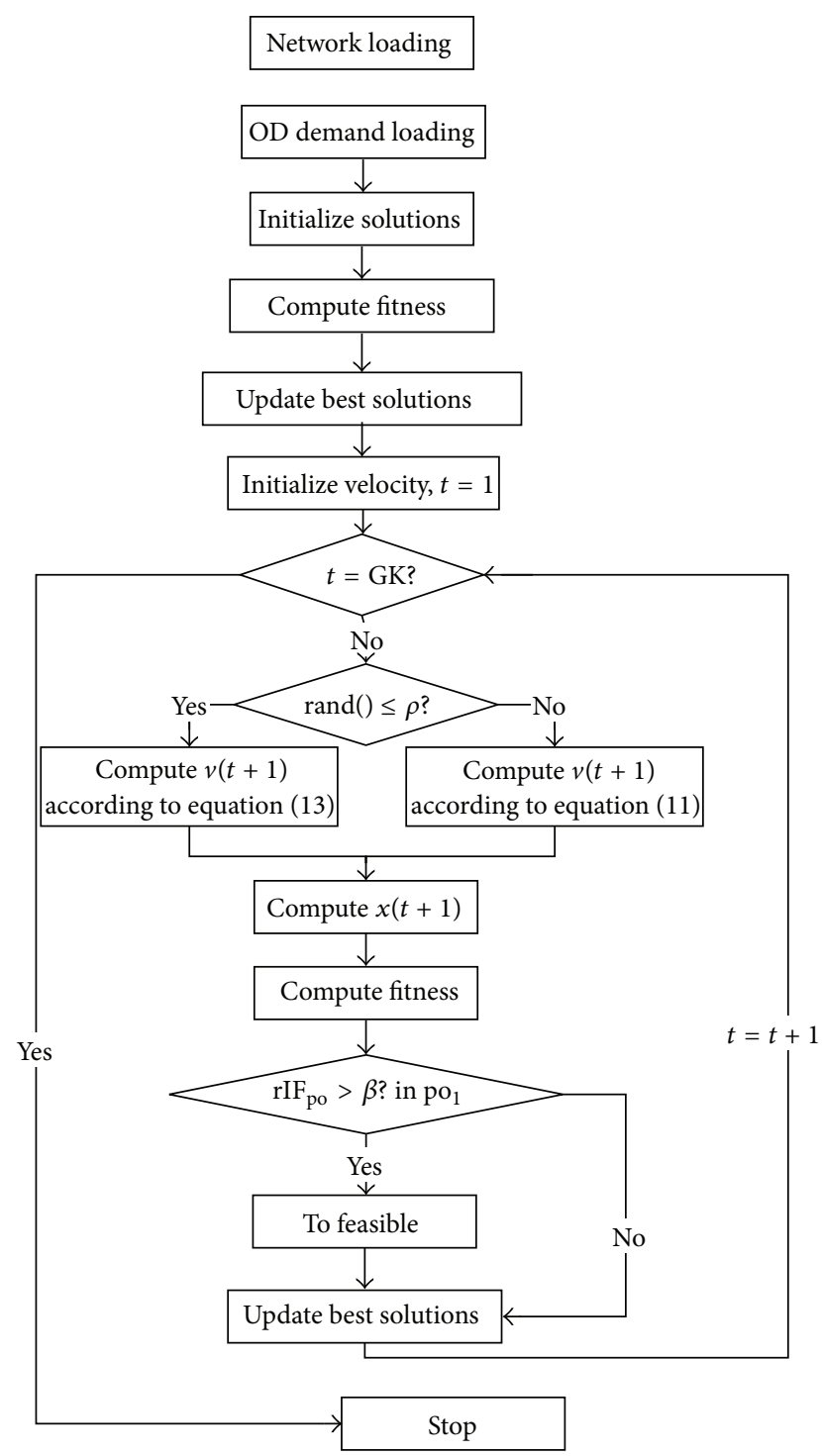

FigURE 3: Flow of solving procedure.

TABLE 2: Parameters of algorithm.

\begin{tabular}{ccccccc}
\hline$\rho$ & $\beta$ & $C 1$ & $C 2$ & $w_{\text {ini }}$ & $w_{\text {end }}$ & Gk \\
\hline 0.5 & 0.95 & 2.1 & 2.1 & 0.9 & 0.1 & 500 \\
\hline
\end{tabular}

Algorithm B. 50 times experiments are carried out by two different algorithms with different sizes of population, respectively. The comparison of ATD, MTD, and average running times with different algorithms are shown in Table 3. The better optimal solution can be obtained by Algorithm A than Algorithm B.

The distribution of optimal solutions under Algorithm A $\left(Q_{\text {po }}=80\right)$ is shown in Figure 4 .

Choose a running; the procedure of convergence of ADT and MDT are shown in Figures 5 and 6, respectively.

5.3. Analyzing the Results of Experiments. An optimal solution of model A is chosen, in which ATD is 194 seconds

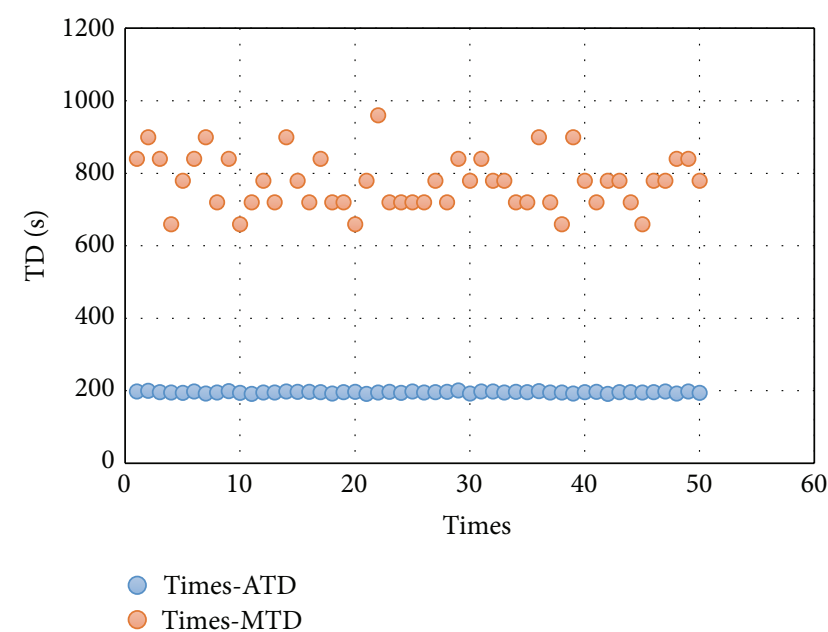

FIGURE 4: Distribution of optimal solutions.

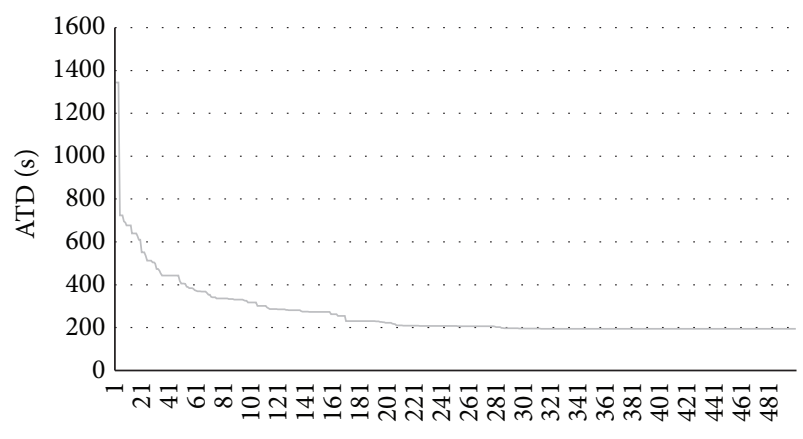

FIgURE 5: Convergence curve of ATD.

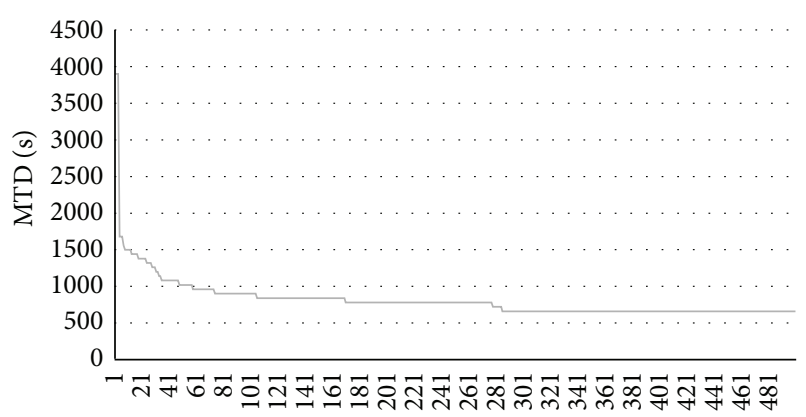

FIGURE 6: Convergence curve of MTD.

and MTD is 660 seconds. The detail of solution is shown in Table 4, where eight time segments during 7:30-8:50 are chosen.

The key indicators of the solutions are compared with the past research which is proposed by Zhao et al. [6]. The model in this paper is named as model A. The model proposed by Zhao et al. [6] is named as model B. Comparison of key indicators under different conditions is shown in Table 5. The key indicators become better under control than the one without control. The value of MDT under control of model 
TABLE 3: Comparison of solution with different algorithms.

\begin{tabular}{lccccccc}
\hline \multirow{2}{*}{ Algorithm } & \multirow{2}{*}{ Number of populations } & \multirow{2}{*}{$Q_{\text {po }}$} & \multicolumn{2}{c}{ Best } & \multicolumn{2}{c}{ Average } & \multirow{2}{*}{ Average running time (seconds) } \\
& 2 & 40 & 193 & 660 & 200 & 812 & 26 \\
Algorithm A & 1 & 80 & 195 & 780 & 432 & 1260 & 26 \\
Algorithm B & 2 & 80 & 192 & 660 & 195 & 775 & 51 \\
Algorithm A & 1 & 160 & 196 & 720 & 292 & 985 & 54 \\
Algorithm B & 2 & & & & & ATD & \\
\hline
\end{tabular}

TABLE 4: Example of optimal solution.

\begin{tabular}{lcccccccc}
\hline Station name & $7: 30-7: 40$ & $7: 40-7: 50$ & $7: 50-8: 00$ & $8: 00-8: 10$ & $8: 10-8: 20$ & $8: 20-8: 30$ & $8: 30-8: 40$ & $8: 40-8: 50$ \\
\hline$S_{1}$ & 2100 & 1660 & 1290 & 1330 & 1490 & 1310 & 1770 & 950 \\
$S_{2}$ & 1260 & 2010 & 1340 & 1700 & 1370 & 1710 & 1810 & 700 \\
$S_{3}$ & 1150 & 1430 & 1900 & 1670 & 2000 & 1770 & 1280 \\
$S_{0}$ & 2100 & 1450 & 1240 & 1810 & 1510 & 1280 & 1300 & 1210 \\
\hline
\end{tabular}

TABLE 5: Comparison of key indicators.

\begin{tabular}{lcccc}
\hline & ADT & MDT & $\max \left(W_{i}\right)$ & $W_{i} \leq \mathrm{PL}_{i}$ \\
\hline Without control & 178 & 900 & 7545 & No \\
Under control of model A & 194 & 660 & 1799 & Yes \\
Under control of model B & 162 & 900 & 7530 & No \\
\hline
\end{tabular}

TABLE 6: Comparison of maximum number of passenger delayed.

\begin{tabular}{lcccc}
\hline & $S_{0}$ & $S_{1}$ & $S_{2}$ & $S_{3}$ \\
\hline Without control & & & & \\
$\quad$ Outside of station & 0 & 0 & 0 & 0 \\
$\quad$ In station & 6214 & 0 & 0 & 0 \\
Under control & & & & \\
$\quad$ Outside of station & 1428 & 1316 & 1652 & 1752 \\
$\quad$ In station & 486 & 0 & 0 & 0 \\
\hline
\end{tabular}

$\mathrm{A}$ is better than that of model B. The maximum number of waiting passengers under control of model $\mathrm{A}$ is less than the safety limitation of station.

The comparison of maximum number of passengers delayed under control against the one without control is shown in Table 6. It can be observed that the large number of passengers waiting next coming trains in $S_{0}$ is shared by other stations through inflow control.

\section{Conclusion}

In this paper, CPICN is proposed considering the safety of stations. The model of CPICN is built with the objective of minimizing ADT and MDT under constraint of capacity of station and train, O-D demand, schedule, and flow conservation. The PSO method for constrained problem is used to solving the model, which integrates the approaches about adjusting infeasible solution to feasible solution and crossing infeasible solution to feasible solution. The numerical experiments are carried out to prove the feasibility and effectiveness of the model and solving algorithm.
For the further research, the solving algorithm will be experimented and applied to the real network. Moreover the route choice about passengers under control measure will be the focus.

\section{Conflict of Interests}

The authors declare that there is no conflict of interests regarding the publication of this paper.

\section{Acknowledgments}

Research is supported by National Key Technology Research and Development Program (2014BAG01B04) from the Ministry of Science and Technology of China, Scientific Research Project of Beijing Education Committee (PXM2015_014212_000023), and Project (I15H00010) from Beijing Municipal Science \& Technology Commission.

\section{References}

[1] W. H. K. Lam, Z. Y. Gao, K. S. Chan, and H. Yang, "A stochastic user equilibrium assignment model for congested transit networks," Transportation Research B: Methodological, vol. 33, no. 5, pp. 351-368, 1999.

[2] Y. Lin, "61 Stations under regular passenger flow control after adjusting the ticket price in Beijing urban mass transit network," Xin Jing Newspaper, January 2015, http://www.bj.xinhuanet .com/bjyw/2015-01/10/c_1113945890.htm.

[3] X.-Y. Xu, J. Liu, H.-Y. Li, and J.-Q. Hu, "Analysis of subway station capacity with the use of queueing theory," Transportation Research C: Emerging Technologies, vol. 38, pp. 28-43, 2014.

[4] W. Xie, Passenger control scheme for urban rail transit transfer station [M.S. thesis], Beijing Jiaotong University, Beijing, China, 2012.

[5] Z. Zheng, J. Xi, and Y. S. He, "Study on collaborative flowlimiting safety control of urban railway station in peak hours," Journal of Safety Science and Technology, vol. 10, pp. 5-10, 2013.

[6] P. Zhao, X. M. Yao, and D. D. Yu, "Cooperative passenger inflow control of urban mass transit in peak hours," Journal of Tongji University, vol. 42, no. 9, pp. 1340-1443, 2014. 
[7] L. H. Liu and L. Jiang, "Research on passenger flow control method of urban rail transit network," Railway Transportation and Economy, vol. 33, no. 5, pp. 51-55, 2011.

[8] Z. Guo and N. H. M. Wilson, "Assessing the cost of transfer inconvenience in public transport systems: a case study of the London Underground," Transportation Research Part A: Policy and Practice, vol. 45, no. 2, pp. 91-104, 2011.

[9] R. Eberhart and J. Kennedy, "A new optimizer using particle swarm theory," in Proceedings of the 6th International Symposium on Micro Machine and Human Science (MHS '95), pp. 3943, IEEE, Nagoya, Japan, October 1995.

[10] Y. H. Shi and R. C. Eberhart, "A modified particle swarm optimizer," in Proceedings of the IEEE International Conference on Evolutionary Computation (ICEC '98), pp. 69-73, Anchorage, Alaska, USA, May 1998.

[11] M. Clerc and J. Kennedy, "The particle swarm-explosion, stability, and convergence in a multidimensional complex space," IEEE Transactions on Evolutionary Computation, vol. 6, no. 1, pp. 58-73, 2002.

[12] E. C. G. Wille, E. Yabcznski, and H. S. Lopes, "Discrete capacity assignment in IP networks using particle swarm optimization," Applied Mathematics and Computation, vol. 217, no. 12, pp. 5338-5346, 2011.

[13] Q. He and L. Wang, "A hybrid particle swarm optimization with a feasibility-based rule for constrained optimization," Applied Mathematics and Computation, vol. 186, no. 2, pp. 1407-1422, 2007.

[14] X. Kou, S. Liu, J. Zhang, and W. Zheng, "Co-evolutionary particle swarm optimization to solve constrained optimization problems," Computers and Mathematics with Applications, vol. 57, no. 11-12, pp. 1776-1784, 2009.

[15] Q. He and L. Wang, "An effective co-evolutionary particle swarm optimization for constrained engineering design problems," Engineering Applications of Artificial Intelligence, vol. 20, no. 1, pp. 89-99, 2007.

[16] S. M. Elsayed, R. A. Sarker, and E. Mezura-Montes, "Selfadaptive mix of particle swarm methodologies for constrained optimization," Information Sciences, vol. 277, pp. 216-233, 2014.

[17] G. Koulinas, L. Kotsikas, and K. Anagnostopoulos, "A particle swarm optimization based hyper-heuristic algorithm for the classic resource constrained project scheduling problem," Information Sciences, vol. 277, pp. 680-693, 2014.

[18] C.-L. Sun, J.-C. Zeng, and J.-S. Pan, "An improved vector particle swarm optimization for constrained optimization problems," Information Sciences, vol. 181, no. 6, pp. 1153-1163, 2011.

[19] Z. Michalewicz, "Do not kill unfeasible individuals," in Proceedings of the 4th Intelligent Information Systems Workshop, pp. 110123, Augustów, Poland, June 1995.

[20] S. O. Kimbrough, G. J. Koehler, M. Lu, and D. H. Wood, "On a Feasible-Infeasible Two-Population (FI-2Pop) genetic algorithm for constrained optimization: distance tracing and no free lunch," European Journal of Operational Research, vol. 190, no. 2, pp. 310-327, 2008.

[21] A. Nuzzolo, U. Crisalli, and L. Rosati, "A schedule-based assignment model with explicit capacity constraints for congested transit networks," Transportation Research Part C: Emerging Technologies, vol. 20, no. 1, pp. 16-33, 2012.

[22] C. O. Tong and S. C. Wong, "A predictive dynamic traffic assignment model in congested capacity-constrained road networks," Transportation Research Part B: Methodological, vol. 34, no. 8, pp. 625-644, 2000.
[23] M. H. Poon, S. C. Wong, and C. O. Tong, "A dynamic schedulebased model for congested transit networks," Transportation Research B: Methodological, vol. 38, no. 4, pp. 343-368, 2004. 


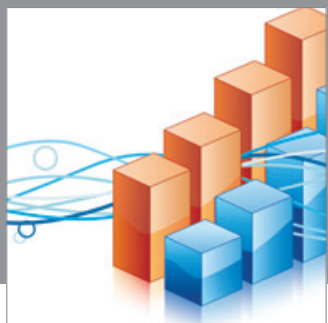

Advances in

Operations Research

mansans

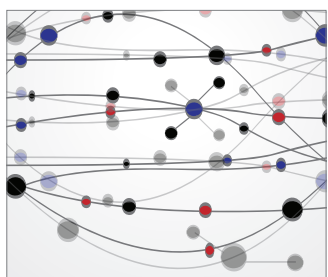

The Scientific World Journal
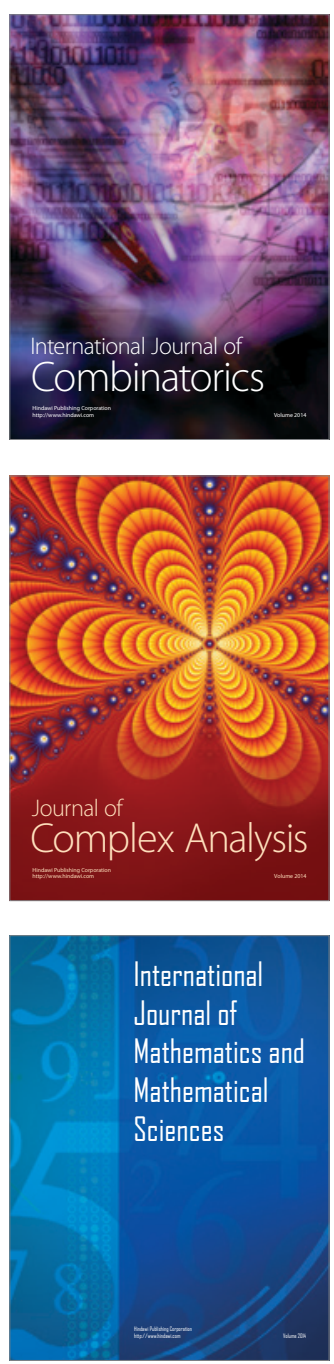
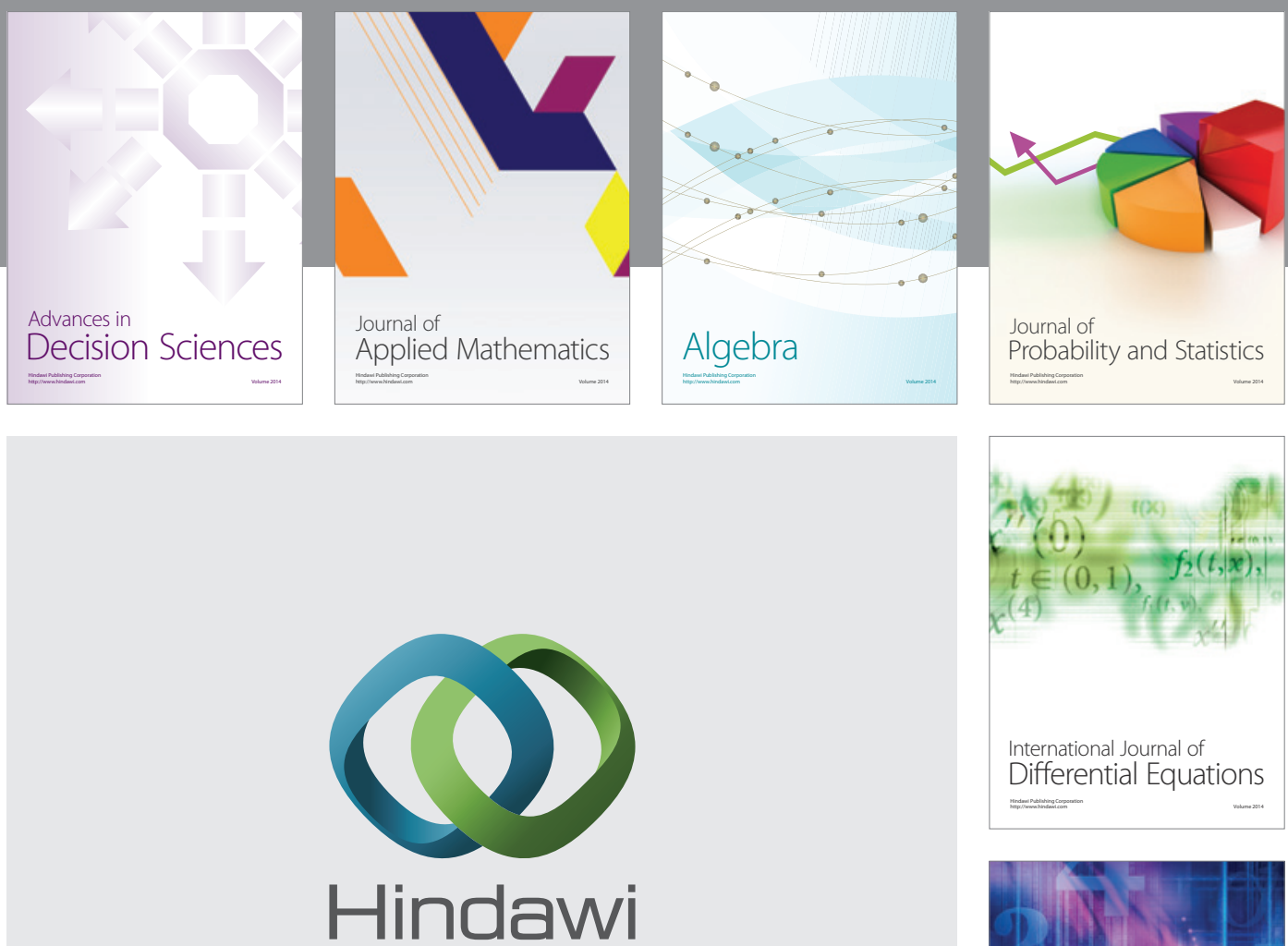

Submit your manuscripts at http://www.hindawi.com
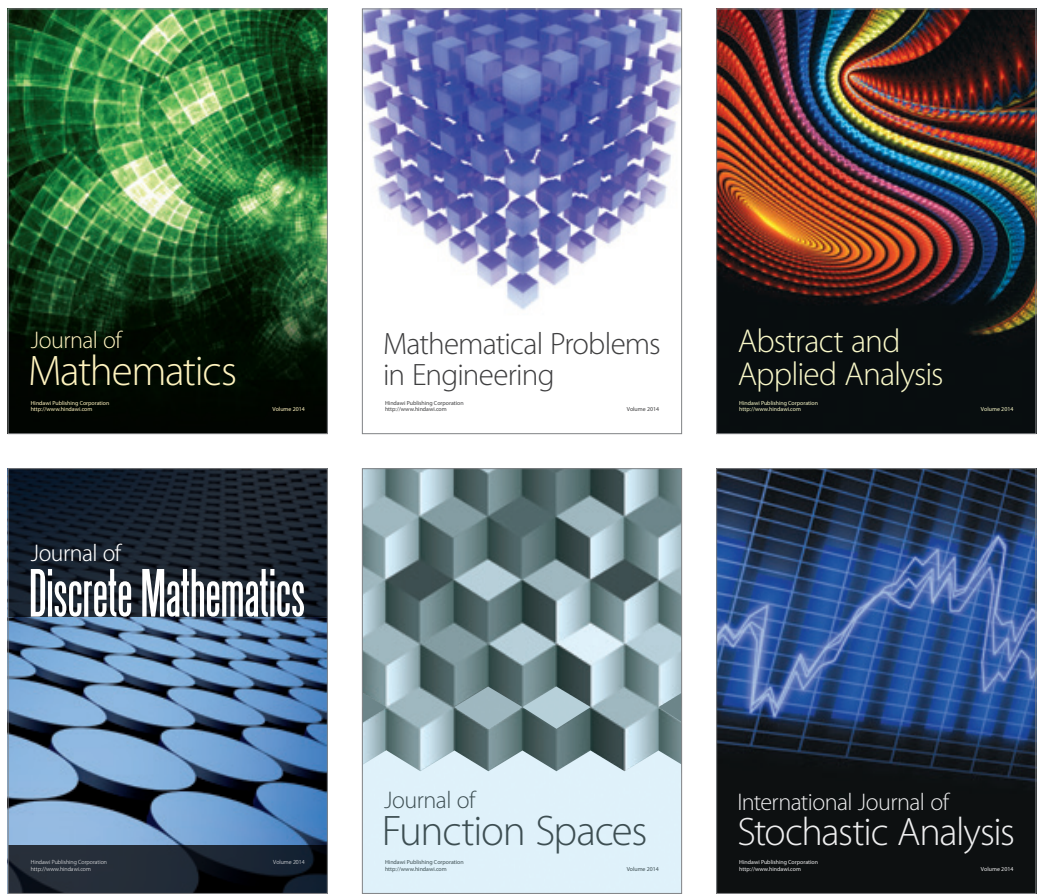

Journal of

Function Spaces

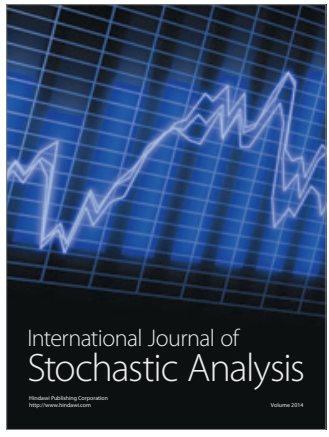

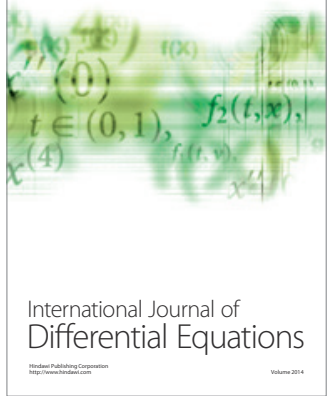
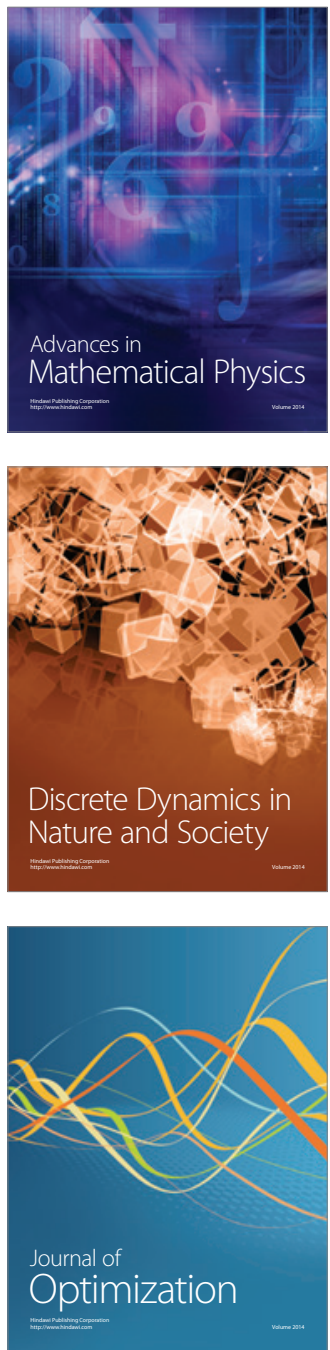